NONCOMMUTATIVE GEOMETRY

AND QUANTUM GROUPS

BANACH CENTER PUBLICATIONS, VOLUME 61

INSTITUTE OF MATHEMATICS

POLISH ACADEMY OF SCIENCES

WARSZAWA 2003

\title{
QUANTUM LENS SPACES AND PRINCIPAL ACTIONS ON GRAPH $C^{*}$-ALGEBRAS
}

\author{
WOJCIECH SZYMAŃSKI \\ School of Mathematical and Physical Sciences \\ The University of Newcastle, Callaghan, NSW 2308, Australia \\ E-mail:wojciech@maths.newcastle.edu.au
}

\begin{abstract}
We study certain principal actions on noncommutative $C^{*}$-algebras. Our main examples are the $\mathbb{Z}_{p^{-}}$and $\mathbb{T}$-actions on the odd-dimensional quantum spheres, yielding as fixedpoint algebras quantum lens spaces and quantum complex projective spaces, respectively. The key tool in our analysis is the relation of the ambient $C^{*}$-algebras with the Cuntz-Krieger algebras of directed graphs. A general result about the principality of the gauge action on graph algebras is given.
\end{abstract}

0. Introduction. Classical complex projective spaces may be defined as orbit spaces of free actions of the circle group on odd-dimensional spheres. This idea can be extended to the noncommutative world. Namely, the $C^{*}$-algebras $C\left(\mathbb{C} P_{q}^{n-1}\right)$ of the quantum complex projective spaces are defined as fixed-point algebras for certain $\mathbb{T}$-actions on the quantum odd-dimensional spheres $C\left(S_{q}^{2 n-1}\right)$ [16]. (Note that according to a recent result of Hawkins and Landi [6], the Vaksman-Soibelman quantum unitary spheres coincide with the quantum orthogonal odd spheres defined by Reshetikhin, Takhtadzhyan and Faddeev [15].) Similarly, classical (generalized) lens spaces may be defined as orbit spaces of free actions of finite cyclic groups on odd-dimensional spheres. This construction was extended to noncommutative setting in [8]. Therein, natural $\mathbb{Z}_{p}$-actions on the odd-dimensional quantum spheres are considered whose fixed-point algebras constitute the $C^{*}$-algebras of continuous functions on the quantum lens spaces. This definition gives rise to algebras which are in general non-isomorphic with the ones constructed earlier by Matsumoto and Tomiyama [12].

In this note, we show that the above-mentioned actions on noncommutative $C^{*}$-algebras are principal in the sense of Ellwood [5]. Special cases include the $\mathbb{T}$-action on $C\left(S U_{q}(2)\right)$ yielding the standard Podleś sphere $C\left(S_{q 0}^{2}\right)[13]$, and the $\mathbb{Z}_{2}$-actions on $C\left(S_{q}^{2 n-1}\right)$ giving rise to the quantum odd-dimensional real projective spaces $C\left(\mathbb{R} P_{q}^{2 n-1}\right)$ [7].

2000 Mathematics Subject Classification: 46L65, 46L55.

The paper is in final form and no version of it will be published elsewhere. 
In order to smoothly handle $C^{*}$-algebraic complications we make use of the machinery of Cuntz-Krieger algebras of directed graphs. In fact, even though the $C^{*}$-algebras under consideration are defined via complicated relations, all of them are isomorphic to certain graph $C^{*}$-algebras $[7,8]$. This makes the determination of their ideal structure and $K$ theoretic invariants a matter of routine calculations (cf. $[7,8]$ ). It also greatly simplifies the proofs of principality of the actions. Indeed, both the $\mathbb{Z}_{p}$-actions giving the $q$-lens spaces and the $\mathbb{T}$-actions giving the quantum complex projective spaces are defined via very simple formulae when transported to the graph algebras. In fact, the latter become nothing else but the canonical gauge actions of the corresponding graph algebras. This last observation motivates our brief discussion of principality of the gauge actions on arbitrary graph algebras.

1. Graph $C^{*}$-algebras. We briefly recall the concept of a graph $C^{*}$-algebra. (For more details we refer the reader to [11] and [2].) Let $E$ be a countable graph with the set of vertices $E^{0}$ and the set of directed edges $E^{1}$. (If $e \in E^{1}$ then $s(e)$ is the source of $e$ and $r(e)$ is its range.) For simplicity sake we assume that every vertex in $E$ emits only finitely many edges. Then $C^{*}(E)$ is defined as the universal $C^{*}$-algebra generated by partial isometries $\left\{S_{e} \mid e \in E^{1}\right\}$ with mutually orthogonal ranges and by projections $\left\{P_{v} \mid v \in E^{0}\right\}$ such that

$$
\begin{aligned}
S_{e}^{*} S_{e} & =P_{r(e)} \text { for each } e \in E^{1}, \\
P_{v} & =\sum_{s(f)=v} S_{f} S_{f}^{*} \text { for each } v \in E^{0} \text { emitting at least one edge. }
\end{aligned}
$$

These graph algebras generalize and contain as a subclass the classical Cuntz-Krieger algebras $[4,3]$.

According to the general Cuntz formula valid for graph algebras (cf. [14, Theorem $3.2]$ ), the $K$-theory of $C^{*}(E)$ can be calculated as follows. Let $E_{+}^{0}$ be the set of those vertices of $E$ that emit at least one edge, and let $\mathbb{Z} E^{0}$ and $\mathbb{Z} E_{+}^{0}$ be the free abelian groups with generators $E^{0}$ and $E_{+}^{0}$, respectively. Let $A_{E}: \mathbb{Z} E_{+}^{0} \rightarrow \mathbb{Z} E^{0}$ be the map defined by

$$
A_{E}(v)=\left(\sum_{s(e)=v} r(e)\right)-v .
$$

Then

$$
K_{0}\left(C^{*}(E)\right) \cong \operatorname{coker}\left(A_{E}\right), \quad K_{1}\left(C^{*}(E)\right) \cong \operatorname{ker}\left(A_{E}\right)
$$

For an arbitrary graph $E$ it is now also possible to determine the ideal structure of $C^{*}(E)$ and, in particular, its primitive ideal space $[1,9]$.

2. Quantum lens spaces. In [16], Vaksman and Soibelman defined and analyzed $q$ analogues of the odd-dimensional spheres, as homogeneous spaces of the quantum special unitary groups of Woronowicz [18]. For $q \in(0,1]$, the $C^{*}$-algebra $C\left(S_{q}^{2 n-1}\right)$ is therein identified with a universal $C^{*}$-algebra generated by $n$ elements $z_{1}, \ldots, z_{n}$, subject to certain relations. In the classical case $q=1$, these generators $\left\{z_{j}\right\}$ are the coordinate functions for $S^{2 n-1} \subset \mathbb{C}^{n}$. Hence the elements of the *-algebra $\operatorname{Pol}\left(S_{q}^{2 n-1}\right)$, generated algebraically by $z_{1}, \ldots, z_{n}$, play the role of polynomials on the quantum sphere $S_{q}^{2 n-1}$. 
As $C^{*}$-algebras, $C\left(S_{q}^{2 n-1}\right)$ are all isomorphic for $q \in(0,1)$. The Vaksman-Soibelman relations make sense for $q=0$ as well and lead to an isomorphic $C^{*}$-algebra.

Imitating the classical construction one can define quantum analogues of (generalized) lens spaces as follows [8]. Choose an integer $p \geq 2$ and integers $m_{1}, \ldots, m_{n}$ relatively prime to $p$. Let $\theta=e^{2 \pi i / p}$. There exists an order $p$ automorphism $\tilde{\Lambda}$ of $C\left(S_{q}^{2 n-1}\right)$ determined by

$$
\tilde{\Lambda}\left(z_{i}\right):=\theta^{m_{i}} z_{i} .
$$

The $C^{*}$-algebra $C\left(L_{q}\left(p ; m_{1}, \ldots, m_{n}\right)\right)$ of continuous functions on the quantum lens space $L_{q}\left(p ; m_{1}, \ldots, m_{n}\right)$ is, by definition, the fixed-point algebra for $\tilde{\Lambda}$ :

$$
C\left(L_{q}\left(p ; m_{1}, \ldots, m_{n}\right)\right):=C\left(S_{q}^{2 n-1}\right)^{\tilde{\Lambda}} .
$$

Thus, if $p=2$ (and necessarily $m_{1}=\cdots=m_{n}=1$ and $\theta=-1$ ) then the such defined quantum lens spaces coincide with the quantum real projective spaces studied in [7]. The polynomial algebra on $L_{q}\left(p ; m_{1}, \ldots, m_{n}\right)$ is defined as the fixed-point algebra of the restriction of $\tilde{\Lambda}$ to $\operatorname{Pol}\left(S_{q}^{2 n-1}\right)$.

3. Relation with graph algebras. The odd-dimensional quantum spheres of Vaksman and Soibelman correspond to graph $C^{*}$-algebras, as follows. Let $L_{2 n-1}$ be the directed graph with vertices $\left\{v_{1}, \ldots, v_{n}\right\}$ and edges $\left\{e_{i, j} \mid i=1, \ldots, n, j=i, \ldots, n\right\}$, with $s\left(e_{i, j}\right)=v_{i}$ and $r\left(e_{i, j}\right)=v_{j}$. An explicit (on generators) isomorphism

$$
\phi: C\left(S_{q}^{2 n-1}\right) \rightarrow C^{*}\left(L_{2 n-1}\right)
$$

is given in [7, Theorem 4.4]. Thus $\Lambda:=\phi \tilde{\Lambda} \phi^{-1}$ is an automorphism of $C^{*}\left(L_{2 n-1}\right)$ such that the fixed-point algebra $C^{*}\left(L_{2 n-1}\right)^{\Lambda}$ is isomorphic via $\phi$ to $C\left(L_{q}\left(p ; m_{1}, \ldots, m_{n}\right)\right)$. This isomorphism allows one to study our quantum lens spaces with the help of the extensively developed machinery of Cuntz-Krieger algebras. In particular, $\Lambda$ is a quasifree automorphism of the graph algebra $C^{*}\left(L_{2 n-1}\right)$ determined by

$$
\Lambda\left(S_{e_{i, j}}\right)=\theta^{m_{i}} S_{e_{i, j}} .
$$

It turns out (cf. [8, Theorem 2.5]) that $C^{*}\left(L_{2 n-1}\right)^{\Lambda}$ (and hence $C\left(L_{q}\left(p ; m_{1}, \ldots, m_{n}\right)\right)$ ) is itself isomorphic to a certain graph algebra. Furthermore, the same is true about the crossed product $C^{*}\left(L_{2 n-1}\right) \rtimes_{\Lambda} \mathbb{Z}_{p}$. Indeed, by virtue of the results of [10], this crossed product is naturally $*$-isomorphic to the $C^{*}$-algebra of the skew-product graph $L_{2 n-1} \times_{c}$ $\mathbb{Z}_{p}$. Here $c$ is a $\mathbb{Z}_{p}$-valued labeling of the edges of $L_{2 n-1}$ such that $c\left(e_{i, j}\right)=m_{i}$. The corresponding skew-product graph has vertices $L_{2 n-1}^{0} \times \mathbb{Z}_{p}$ and edges $L_{2 n-1}^{1} \times \mathbb{Z}_{p}$, with $s\left(e_{i, j}, m\right)=\left(v_{i}, m-m_{i}\right)$ and $r\left(e_{i, j}, m\right)=\left(v_{j}, m\right)$. Since the action $\Lambda$ is saturated [8], the fixed-point algebra and the crossed product are strongly Morita equivalent. Consequently,

$$
C\left(L_{q}\left(p ; m_{1}, \ldots, m_{n}\right)\right) \cong_{M} C^{*}\left(L_{2 n-1} \times_{c} \mathbb{Z}_{p}\right) .
$$

4. Principal actions. As expected, the $\mathbb{Z}_{p}$-action $\tilde{\Lambda}$ defining our quantum lens spaces has nice properties resembling those of its classical counterpart. In particular, it is principal in the sense of Ellwood [5]. In the present article we only consider actions of compact groups, which are automatically proper. Thus an action of a compact group 
$\Gamma$ on a $C^{*}$-algebra $\mathcal{A}$ is principal in the sense of $[5$, Definition 2.4] if and only if the linear map

$$
\Phi: \mathcal{A} \otimes_{\text {alg }} \mathcal{A} \rightarrow \mathcal{A} \otimes C(\Gamma), \quad \Phi: x \otimes y \mapsto(x \otimes I) \delta(y),
$$

has norm-dense range. Here $\delta: \mathcal{A} \rightarrow \mathcal{A} \otimes C(\Gamma)$ is the corresponding coaction.

Proposition 1. The action $\tilde{\Lambda}$ of $\mathbb{Z}_{p}$ on $C\left(S_{q}^{2 n-1}\right)$ defined by (5) is principal.

Proof. It sufficies to prove that the action $\Lambda$ of $\mathbb{Z}_{p}$ on $C^{*}\left(L_{2 n-1}\right)$ defined by (8) is principal. The corresponding coaction $\delta: C^{*}\left(L_{2 n-1}\right) \rightarrow C^{*}\left(L_{2 n-1}\right) \otimes C\left(\mathbb{Z}_{p}\right)$ is given on the generators by

$$
\delta\left(S_{e_{i, j}}\right)=S_{e_{i, j}} \otimes \chi^{m_{i}}
$$

where $\chi$ is the character of $\mathbb{Z}_{p}$ such that $\chi(d)=\theta^{d}$. Since $\theta$ is a $p^{\text {th }}$ primitive root of unity, this unitary element $\chi$ generates the algebra $C\left(\mathbb{Z}_{p}\right)$ of functions on $\mathbb{Z}_{p}$.

For each $i=1, \ldots, n$ and $k=1,2, \ldots$ we have

$$
\Phi\left(S_{e_{i, i}}^{* k} \otimes S_{e_{i, i}}^{k}\right)=\left(S_{e_{i, i}}^{* k} \otimes I\right)\left(S_{e_{i, i}}^{k} \otimes \chi^{k m_{i}}\right)=P_{v_{i}} \otimes \chi^{k m_{i}} .
$$

Since $m_{i}$ is relatively prime to $p$, by assumption, (12) implies that the range of $\Phi$ contains $P_{v_{i}} \otimes C\left(\mathbb{Z}_{p}\right)$. Thus, it also contains $I \otimes C\left(\mathbb{Z}_{p}\right)$, since in $C^{*}\left(L_{2 n-1}\right)$ we have $I=\sum_{i} P_{v_{i}}$. Consequently, the map $\Phi$ is surjective.

If $m_{1}=\cdots=m_{n}=1$ then the action $\Lambda$ coincides with the restriction of the gauge action to the group of $p^{\text {th }}$ roots of unity. Recall that for an arbitrary graph $E$ the gauge action $\gamma: \mathbb{T} \rightarrow \operatorname{Aut}\left(C^{*}(E)\right)$ is defined by

$$
\gamma_{t}\left(S_{e}\right)=t S_{e}, \quad \gamma_{t}\left(P_{v}\right)=P_{v}
$$

for all $e \in E^{1}, v \in E^{0}$ and $t \in \mathbb{T} \subset \mathbb{C}$. The $\mathbb{T}$-action on $C\left(S_{q}^{2 n-1}\right)=C^{*}\left(z_{1}, \ldots, z_{n}\right)$ such that $t \cdot z_{i}=t z_{i}$ yields the fixed-point algebra $C\left(\mathbb{C} P_{q}^{n-1}\right)$ [16]. Under the isomorphism $C\left(S_{q}^{2 n-1}\right) \cong C^{*}\left(L_{2 n-1}\right)$ of $[7$, Theorem 4.4] this action is transported into the gauge action $\gamma$ on $C^{*}\left(L_{2 n-1}\right)$. This observation motivates our next proposition.

Proposition 2. If $E$ is a directed graph such that each vertex emits finitely many edges, each vertex emits at least one edge and receives at least one, then the gauge action $\gamma: \mathbb{T} \rightarrow \operatorname{Aut}\left(C^{*}(E)\right)$ is principal.

Proof. Let $z$ denote the canonical generator of $C(\mathbb{T})$. Then the coaction $\delta: C^{*}(E) \rightarrow$ $C^{*}(E) \otimes C(\mathbb{T})$, corresponding to the gauge action $\gamma$, is defined by

$$
\delta\left(S_{e}\right)=S_{e} \otimes z, \quad \delta\left(P_{v}\right)=P_{v} \otimes I
$$

for all $e \in E^{1}, v \in E^{0}$. Since the powers of $z$ span a dense subspace of $C(\mathbb{T})$ and finite sums of projections of the form $P_{v}, v \in E^{0}$, give rise to an approximate unit for $C^{*}(E)$, it sufficies to show that the image of the map $\Phi$ (defined in (10)) contains $P_{v} \otimes z^{k}$ for each $v \in E^{0}, k \in \mathbb{Z}$. To this end, fix a vertex $v$ and a positive integer $k$. Since each vertex of $E$ receives at least one edge it follows that there exists a path $\alpha=\alpha_{1} \cdots \alpha_{k}$, with $\alpha_{i} \in E^{1}$, which ends in $v$. For $S_{\alpha}=S_{\alpha_{1}} \cdots S_{\alpha_{k}}$ we have

$$
\Phi\left(S_{\alpha}^{*} \otimes S_{\alpha}\right)=\left(S_{\alpha}^{*} \otimes I\right) \delta\left(S_{\alpha}\right)=\left(S_{\alpha}^{*} \otimes I\right)\left(S_{\alpha} \otimes z^{k}\right)=P_{v} \otimes z^{k} .
$$


Since each vertex of $E$ emits finitely many edges and at least one, an inductive application of (2) yields

$$
P_{v}=\sum_{s(\beta)=v,|\beta|=k} S_{\beta} S_{\beta}^{*} .
$$

The summation in (16) extends over all paths of length $k$ in $E$ which begin at $v$. Thus we have

$$
\Phi\left(\sum_{s(\beta)=v,|\beta|=k} S_{\beta} \otimes S_{\beta}^{*}\right)=\sum_{s(\beta)=v,|\beta|=k}\left(S_{\beta} \otimes I\right)\left(S_{\beta}^{*} \otimes z^{-k}\right)=P_{v} \otimes z^{-k} .
$$

Combining (14), (15) and (17) we conclude that $\Phi$ satisfies the required property.

On the other hand, one can show that if $E$ contains a vertex which does not emit any edges then the gauge action is not principal.

Corollary 3. The $\mathbb{T}$-action on $C\left(S_{q}^{2 n-1}\right)=C^{*}\left(z_{1}, \ldots, z_{n}\right)$ such that $t \cdot z_{i}=t z_{i}$, whose fixed-point algebra is $C\left(\mathbb{C} P_{q}^{n-1}\right)[16]$, is principal.

Proof. Combine Proposition 2 above with [7, Theorem 4.4 and $\S 4.3]$.

In the case $n=2$, the $C^{*}$-algebra $C\left(S_{q}^{3}\right) \cong C\left(S U_{q}(2)\right)$ is isomorphic to $C^{*}\left(L_{3}\right)$, corresponding to the following graph (cf. $[17,7])$ :

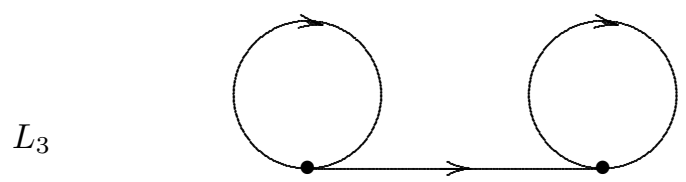

The gauge action $\gamma$ on $C^{*}\left(L_{3}\right)$ is principal, with the fixed-point algebra isomorphic to the minimal unitization of the compacts. Differently interpreted, this setting corresponds on one hand to the quantum bundle $\mathbb{T} \rightarrow S_{q}^{3} \rightarrow \mathbb{C} P_{q}^{1}$ of [16], and on the other hand to $\mathbb{T} \rightarrow S U_{q}(2) \rightarrow S_{q 0}^{2}$ of [13].

Acknowledgements. I would like to thank Piotr M. Hajac for several very useful conversations on principal actions and other topics. I am grateful to the European Commission for partial support of my travel to Warsaw for this school/conference. It is a pleasure to thank Max-Planck-Institut für Mathematik (Bonn) and the Research Grants Committee (Newcastle) for their financial support. I would also like to thank Mathematisches Forschungsinstitut Oberwolfach, where this note was completed during my stay under the Research-in-Pairs programme.

\section{References}

[1] T. Bates, J. H. Hong, I. Raeburn and W. Szymański, The ideal structure of the $C^{*}$-algebras of infinite graphs, Illinois J. Math., to appear.

[2] T. Bates, D. Pask, I. Raeburn and W. Szymański, The $C^{*}$-algebras of row-finite graphs, New York J. Math. 6 (2000), 307-324.

[3] J. Cuntz, A class of $C^{*}$-algebras and topological Markov chains II: Reducible chains and the Ext-functor for $C^{*}$-algebras, Invent. Math. 63 (1981), 25-40. 
[4] J. Cuntz and W. Krieger, A class of $C^{*}$-algebras and topological Markov chains, Invent. Math. 56 (1980), 251-268.

[5] D. A. Ellwood, A new characterisation of principal actions, J. Funct. Anal. 173 (2000), 49-60.

[6] E. Hawkins and G. Landi, Fredholm modules for quantum Euclidean spheres, preprint, 2002.

[7] J. H. Hong and W. Szymański, Quantum spheres and projective spaces as graph algebras, Commun. Math. Phys. 232 (2002), 157-188.

[8] J. H. Hong and W. Szymański, Quantum lens spaces and graph algebras, Pacific J. Math., to appear.

[9] J. H. Hong and W. Szymański, The primitive ideal space of the $C^{*}$-algebras of infinite graphs, J. Math. Soc. Japan, to appear.

[10] A. Kumjian and D. Pask, $C^{*}$-algebras of directed graphs and group actions, Ergodic Theory \& Dynamical Systems 19 (1999), 1503-1519.

[11] A. Kumjian, D. Pask, I. Raeburn and J. Renault, Cuntz-Krieger algebras of directed graphs, J. Funct. Anal. 144 (1997), 505-541.

[12] K. Matsumoto and J. Tomiyama, Noncommutative lens spaces, J. Math. Soc. Japan 44 (1992), 13-41.

[13] P. Podleś, Quantum spheres, Lett. Math. Phys. 14 (1987), 193-202.

[14] I. Raeburn and W. Szymański, Cuntz-Krieger algebras of infinite graphs and matrices, Trans. Amer. Math. Soc., to appear.

[15] N. Yu. Reshetikhin, L. A. Takhtadzhyan and L. D. Faddeev, Quantization of Lie groups and Lie algebras, Algebra i Analiz 1 (1989), 178-206 (in Russian); translation in Leningrad Math. J. 1 (1990), 193-225.

[16] L. L. Vaksman and Y. S. Soibelman, Algebra of functions on quantum $S U(n+1)$ group and odd dimensional quantum spheres, Algebra i Analiz 2 (1990), 101-120.

[17] S. L. Woronowicz, Twisted SU(2) group. An example of a noncommutative differential calculus, Publ. Res. Inst. Math. Sci. 23 (1987), 117-181.

[18] S. L. Woronowicz, Tannaka-Krein duality for compact matrix pseudogroups. Twisted $S U(N)$ groups, Invent. Math. 93 (1988), 35-76. 